\title{
BMJ Open Nutrition interventions for children aged less than 5 years following natural disasters: a systematic review protocol
}

\author{
Pranil Man Singh Pradhan, Rolina Dhital, Huma Subhani
}

To cite: Pradhan PMS, Dhital $R$, Subhani $H$. Nutrition interventions for children aged less than 5 years following natural disasters: a systematic review protocol. BMJ Open 2015;5:e009525. doi:10.1136/bmjopen-2015009525

- Prepublication history for this paper is available online. To view these files please visit the journal online (http://dx.doi.org/10.1136/ bmjopen-2015-009525).

Received 24 July 2015 Revised 9 November 2015 Accepted 9 November 2015

CrossMark

Department of Community Health Sciences, Patan Academy of Health Sciences, Lalitpur, Nepal

\section{Correspondence to} Dr Pranil Man Singh Pradhan; pranil.pradhan@gmail.com

\section{ABSTRACT}

Introduction: Malnutrition among children is a serious public health problem in the aftermath of any natural disaster. We will review the various nutrition interventions for children aged $<5$ years in countries where natural disasters occurred and analyse the effect on nutrition-related outcomes.

Methods and analysis: We will conduct a systematic review on nutrition intervention studies following natural disasters that were published between January 2000 and December 2015. Study selection will follow the Preferred Reporting Items for Systematic Reviews and Meta-Analyses (PRISMA) guidelines. The Cochrane Risk of Bias $(\mathrm{RoB})$ tool will be used for randomised controlled trials and Risk of Bias Assessment for Non-Randomized Studies (RoBANS) will be used for non-randomised studies. The quality of evidence will be assessed using Grading of Recommendations Assessment, Development, and Evaluation (GRADE) guidelines. If sufficient data are available, we will conduct meta-analyses to establish the relationship between nutrition interventions and nutrition outcome indicators. All statistical analyses will be performed using Review Manager (Rev Man) V.5.3 for Windows. Heterogeneity of the data will be tested using the standard $\chi^{2}$ test. A fixed-effect model will be used for the studies with high heterogeneity $(p$ value $>0.10$, $\mathrm{I}^{2} \leq 50 \%$ ). For dichotomous and continuous data, relative risk (RR) and mean difference with $95 \% \mathrm{Cl}$ will be used respectively. Subgroup analysis will be performed for studies with low heterogeneity ( $p$ value $\leq 0.10)$. We will use $Z$ score with the level of significance set at $p$ value $<0.05$ to test the total effect. Funnel plots will be used to detect publication bias.

Ethics and dissemination: As primary data will not be collected, formal ethical approval will not be required. The results will be disseminated by publication in peer-reviewed journals, conference presentations and the media.

Registration details: International Prospective Register for Systematic Reviews (PROSPER0) number CRD42015023243 was registered on 1 June 2015.

\section{INTRODUCTION}

Natural disasters occur frequently in developed as well as developing countries. In the

\section{Strengths and limitations of this study}

- The study will be relevant to the current circumstances where natural disasters have put the nutrition status of children at risk.

- It will provide current evidence on effectiveness of nutrition interventions for children aged $<5$ years following natural disasters.

- Quality studies with comparable outcomes may not be available for meta-analysis.

- Inclusion of studies published only in the English language may lead to language bias.

past 15 years, we have witnessed many natural disasters such as the Gujarat earthquake in 2001, the European heat wave in 2003, the Indian Ocean earthquake and tsunami in 2004, the Kashmir earthquake in 2005, Cyclone Nargis in 2008, the Wenchuan earthquake in 2008, and the Haiti earthquake in 2010. ${ }^{1}$ Recently, a devastating earthquake of magnitude 7.8 struck Nepal on 25 April 2015. Natural disasters can result in the deaths of a large number of people and cause significant economic damage. In addition to the immediate impact of natural disasters, the affected populations are susceptible to indirect longterm health issues. People affected by natural disasters are likely to be in a state of psychological stress and fatigue. Moreover, substandard sanitation, inadequate water supplies and poor hygiene make the affected people prone to various diseases. ${ }^{2}$ This, coupled with various compounding factors, such as interruption in food supply and restricted health access, can prove detrimental to vulnerable populations, especially children. ${ }^{3} 4$

Food and nutrition insecurity may arise from any natural disaster, such as an earthquake, drought, flood, cyclone, tsunami, hurricane or tornado. This can lead to a rise in mortality due to acute malnutrition contributing to a state of nutrition emergency. ${ }^{5}$ Natural disasters cause destruction of houses, loss of stored foodgrains, death of livestock and exposure of people living in temporary 
shelters to harsh weather conditions. Moreover, mass destruction of food reserves by natural disasters severely affects the nutritional status of new mothers and also deprives them of a supportive environment to breastfeed. This results in acute malnutrition in newborns and a lack of timely nutritional intervention may lead to long-term consequences such as stunting. ${ }^{6}$ Multiple studies worldwide have shown that natural disasters have negative effects on the growth of children. ${ }^{6-8}$ In countries where the rate of stunting is already high, such events have further aggravated the risk of malnutrition in newborns and growing children.

Prior research on the effects of nutrition interventions following a natural disaster has focused on specific disasters. For example, a nutritional intervention study on complementary feeding in the Wenchuan county of China showed that malnutrition among children decreased significantly 1.5 years after nutrition intervention. ${ }^{9}$ Similarly, following Cyclone Sidr in Bangladesh, the provision of micronutrient powder significantly reduced the rates of stunting among children. ${ }^{10}$ The nutrition rehabilitation programme in Jaffna district of Sri Lanka following the tsunami reduced the rates of Global Acute Malnutrition (GAM) ${ }^{11}$

Despite the frequent occurrence of natural disasters, there is insufficient compiled research evidence of different nutrition interventions. The objective of this study is to review various nutrition interventions for children aged $<5$ years in countries following natural disasters and to analyse the effect that specific nutrition interventions have on nutrition-related outcomes.

\section{METHODS AND ANALYSIS}

We will conduct this systematic review on the nutrition intervention studies that were performed only following natural disasters. In this study, we have defined disaster as 'a serious disruption of the functioning of a community or a society involving widespread human, material, economic or environmental losses and impacts, which exceeds the ability of the affected community or society to cope using its own resources'. ${ }^{12}$ We will consider the disasters contributed by natural hazards such as earthquakes, volcanic activity, landslides, tsunamis, tropical cyclones and other severe storms, tornadoes and high winds, river floods and coastal flooding, wildfires and associated haze, drought, sandstorm/dust storm and infestations. ${ }^{13}$ This protocol is developed on the basis of population, intervention, comparators and outcomes (PICO) questions. Our population will be defined as children aged $<5$ years from areas following natural disasters. The comparators will include the comparison groups of the intervention studies. The outcomes will be focused on anthropometric indicators and anaemia.

\section{Nutrition interventions and nutrition-related outcomes}

Nutrition intervention is defined as any type of intervention for children aged $<5$ years in order to improve the overall nutrition status. The interventions may include food fortification, supplementation, or behavioural and regulatory interventions, which have an impact on nutrition outcomes. ${ }^{14}$ Primary nutrition-related outcomes will be stunting, wasting and underweight. Children with height-for-age Z-score below -2SD from the median of the WHO reference population will be defined as stunted. Children with weight-for-height Z-score below $-2 \mathrm{SD}$ will be defined as wasted. Children with weightfor-age Z-score below -2SD will be defined as underweight. Secondary nutrition-related outcome will be anaemia, defined as haemoglobin concentration below $11 \mathrm{~g} / \mathrm{L}$, among children aged $<5$ years. ${ }^{15}$

\section{Study designs}

Randomised controlled trials (RCT) and cluster RCTs will be included to measure the effectiveness of the nutrition interventions. If such studies are not found on the basis of our PICO questions, longitudinal studies, case-control studies and cross-sectional studies will also be included in the review. Quantitative studies will be independently reviewed by the three reviewers and reported according to the PRISMA guidelines. ${ }^{16}$

\section{Data sources for existing review}

Three researchers will independently search for any existing review or submitted protocol listed in the Cochrane Library or the Cochrane Database of Systematic Reviews (CDSR), Database of Abstracts of Reviews of Effects (DARE), National Institute for Health and Care Excellence (NICE), Educational Resources and Information Center (ERIC) and Campbell library of systematic reviews databases.

\section{Data collection}

Before the process of selection of studies, a consensus will be built on the screening procedure by discussion among the reviewers. Three reviewers (PMSP, RD and HS) will independently search for the titles and abstracts and select the studies that are relevant. All the records of the studies will be managed electronically by the Mendeley Desktop software V.1.14. All three reviewers will read the titles, abstracts and full texts of the selected studies to verify that they match the inclusion criteria. None of the reviewers will be blinded to the name of the authors, affiliations or institutions and journal of publication. If necessary, authors of the selected studies will be contacted for clarification. Any disagreement among the reviewers will be discussed and consensus will be reached. The procedure of study selection will be conducted according to the PRISMA guidelines. ${ }^{17}$

\section{Registration details}

We have registered the protocol of our systematic review on 1 June 2015 at PROSPERO. The registration number for this review is CRD42015023243 available at http:// www.crd.york.ac.uk/PROSPERO/display_record.asp?ID= CRD42015023243\#.VZIYmKZqbxY. 


\section{Search strategy}

The databases that will be searched are PubMed/ MEDLINE, Cumulative Index to Nursing and Allied Health Literature (CINAHL), and Health Internetwork Access to Research Initiative (HINARI). The Boolean method for searches will use key words: nutrition intervention, natural disasters, under five children, under 5, under-5, acute malnutrition, stunting, wasting, underweight, anemia. The initial search strategy in PubMed will be as follows:

( ( ( ( ( ( ( ( (Randomized controlled trial) $)$ OR Cluster randomized controlled trial) AND nutritional intervention) OR nutrition intervention) AND natural disaster) AND under five) OR under 5) OR under-5) AND acute malnutrition) OR stunting) OR wasting) OR underweight) OR anemia).

We will also search for grey literature from the WHO databases and the related links, Emergency Nutrition Network (ENN) database, Global nutrition cluster, UN system of standing committee on nutrition, United Nations Children's Fund (UNICEF), and United Nations Office for the Coordination of Humanitarian Affairs (OCHA). We will search for other published literature identified through the references from the sources aforementioned.

\section{Inclusion and exclusion criteria}

Studies conducted among the children aged 6-59 months following a natural disaster will be included. Studies published in the English language from January 2000 to December 2015 will be included. We will only include quantitative study designs that have a clear intervention.

Interventions that were indirectly related to nutrition outcomes will be excluded from the review. We will exclude any other humanitarian crisis not directly related to natural disasters.

\section{Risk of bias (quality) assessment}

We will use the Cochrane Risk of Bias (RoB) tool for RCT and Risk of Bias Assessment for Non-Randomized Studies (RoBANS) for non-randomised studies. ${ }^{18}{ }^{19}$ We will grade the quality of evidence using the Grading of Recommendations Assessment, Development, and Evaluation (GRADE) guidelines. ${ }^{20-22}$ The three reviewers will carefully assess the included studies independently and agree on the final grading. The evidence will be categorised as very strong, strong, moderate and weak on the basis of the study design, strengths and limitations, population size and effect size of pooled results.

\section{Ethics and dissemination}

Since primary data will not be collected, formal ethical approval will not be required. The results will be disseminated through peer-reviewed publications, conference presentations and the media.

\section{Data synthesis and analysis}

The final review will include a narrative synthesis that will focus on the following: study setting and population, type of natural disaster, nutrition interventions, study design of included studies, sampling methods, sample size, duration of the intervention, effectiveness of interventions, and bias.

If sufficient data are available, we will conduct a metaanalysis to establish the relationship between the nutrition intervention and the aforementioned nutrition outcomes. All statistical analyses will be performed using Review Manager (Rev Man) V.5.3 for Windows. Heterogeneity of the data will be tested using the standard $\chi^{2}$ test. A fixedeffect model will be used for the studies with high heterogeneity ( $\mathrm{p}$ value $>0.10, \mathrm{I}^{2} \leq 50 \%$ ). For dichotomous and continuous data, relative risk (RR) and mean difference with $95 \%$ CI will be used respectively. Subgroup analysis will be performed for studies with low heterogeneity $(p \leq 0.10)$. Total effect will be tested using $\mathrm{Z}$ score with the level of significance set at $p$ value $<0.05$. Funnel plots will be used to detect publication bias.

\section{DISCUSSION}

Almost half of all deaths in children are associated with undernutrition and this could increase following natural disasters. ${ }^{23}$ Furthermore, natural disasters often unmask pre-existing nutrition status particularly in low-income settings that could be well above the emergency threshold. ${ }^{24}$ Nutrition actions in emergencies are focused on reducing the levels of global acute malnutrition, preventing micronutrient deficiencies, promoting infant and young child feeding, and ensuring adequate access to safe and nutritious food. ${ }^{24}$

However, there is still lack of evidence on what type of nutrition intervention is the most effective following natural disasters. Moreover, natural disasters in lowincome countries are more likely to cause higher morbidity and mortality compared to middle-income and high-income countries due to higher vulnerabilities of the population, weaker healthcare system, and limited surge capacity. ${ }^{12}$ This review should offer evidence-based recommendations on nutrition interventions for healthcare providers working in areas affected by natural disasters.

This systematic review is likely to be limited by methodological challenges. Systematic reviews of RCTs are considered to be the highest level of evidence. However, such trials are difficult to implement in the setting of natural disasters due to ethical issues. ${ }^{12}$ Thus, we may not be able to find adequate trials for this review. Another difficulty will be to find studies with a valid control group to estimate the effect attributable to the nutrition intervention alone. ${ }^{25}$

\section{CONCLUSION}

This systematic review will provide current evidence on various nutrition interventions for children who are 
most vulnerable to malnutrition following natural disasters. The evidence generated from this paper may prove beneficial in terms of nutrition policy and planning in those countries prone to natural disasters.

Acknowledgements The authors acknowledge Brian C Beard, PhD, Visiting Faculty, Department of Biochemistry, Patan Academy of Health Sciences, for language editing.

Contributors RD conceptualised the research. PMSP, RD and HS planned and designed the study protocol. PMSP and RD wrote the first draft. HS revised the final draft of the manuscript. All authors have approved and contributed to the final version of the manuscript.

Competing interests None declared.

Provenance and peer review Not commissioned; externally peer reviewed.

Open Access This is an Open Access article distributed in accordance with the Creative Commons Attribution Non Commercial (CC BY-NC 4.0) license, which permits others to distribute, remix, adapt, build upon this work noncommercially, and license their derivative works on different terms, provided the original work is properly cited and the use is non-commercial. See: http:// creativecommons.org/licenses/by-nc/4.0/

\section{REFERENCES}

1. Provost C. A decade of disasters-get the key data. Guard 2011. http://www.theguardian.com/global-development/datablog/2011/mar/ 18/world-disasters-earthquake-data

2. UNICEF. Water, Sanitation and Hygiene Emergency WASH. http:// www.unicef.org/wash/index_emergency.html (accessed $28 \mathrm{Sep}$ 2015).

3. Datar A, Liu J, Linnemayra S, et al. The impact of natural disasters on child health and investments in Rural India. Soc Sci Med 2013;76:83-91.

4. Bassett L. Nutrition security in Haiti: Pre- and post earthquake conditions and the way forward. World Bank En Breve Series No. 157 2010. http://siteresources.worldbank.org/INTLAC/Resources/ 257803-1269390034020/EnBreve_157_English_Web.pdf

5. The Johns Hopkins and the International Federation of Red Cross and Red Crescent Societies. Public health guide for emergencies. 442-85. http://www.jhsph.edu/research/centers-and-institutes/centerfor-refugee-and-disaster-response/publications_tools/publications/_ CRDR_ICRC_Public_Health_Guide_Book/Chapter_9_Food_and_ Nutrition.pdf (accessed 16 Jul 2015)

6. Pörtner CC. Natural Hazards and Child Health. 2010. http://papers.ssrn. $\mathrm{com} /$ sol3/papers.cfm?abstract_id=1599432 (accessed 20 Jul 2015).

7. Hoddinott J, Kinsey B. Child growth in the time of drought. Oxf Bull Econ Stat 2001;63:409-36.

8. Mahapatra A, Geddam JJ, Marai N, et al. Nutritional status of preschool children in the drought affected Kalahandi district of Orissa. Indian J Med Res 2000;111:90-4.
9. Dong C, Ge P, Ren X, et al. Prospective study on the effectiveness of complementary food supplements on improving status of elder infants and young children in the areas affected by Wenchuan earthquake. PLOS ONE 2013;8:e72711.

10. Rah JH, de Pee S, Halati S, et al. Provision of micronutrient powder in response to the Cyclone Sidr emergency in Bangladesh: crosssectional assessment at the end of the intervention. Food Nutr Bull 2011;32:277-85.

11. Jayatissa R, Bekele A, Kethiswaran A, et al. Community-based management of severe and moderate acute malnutrition during emergencies in Sri Lanka: challenges of implementation. Food Nutr Bull 2012;33:251-60.

12. Gerdin M, Clarke M, Allen C, et al. Optimal evidence in difficult settings: Improving health interventions and decision making in disasters. PLoS Med 2014;11:e1001632.

13. WHO. Definitions: emergencies. http://www.who.int/hac/about/ definitions/en/ (accessed 30 Oct 2015).

14. WHO. Nutrition interventions. e-Library Evid. Nutr. Actions. http:// www.who.int/elena/intervention/en/ (accessed 28 Sep 2015).

15. WHO. Hemoglobin concentrations for the diagnosis of anaemia and assessment of severity. Vitam. Miner. Nutr. Inf. Syst. http://www. who.int/vmnis/indicators/haemoglobin.pdf (accessed 28 Sep 2011).

16. Liberati A, Altman DG, Tetzlaff $\mathrm{J}$, et al. The PRISMA statement for reporting systematic reviews and meta-analyses of studies that evaluate healthcare interventions: explanation and elaboration. $B M J$ 2009;339:b2700. http://www.bmj.com/content/339/bmj.b2700 http:// dx.doi.org/10.1136/bmj.b2700

17. PRISMA. PRISMA 2009 Flow Diagram. Prism. Statement. http:// www.prisma-statement.org/2.1.4-PRISMAFlow2009Diagram.pdf (accessed 4 Oct 2015).

18. Higgins JP, Green S, eds. The Cochrane Collaboration's tool for assessing risk of bias. In: Cochrane handbook for systematic reviews of interventions. 2011. http://handbook.cochrane.org/ chapter_8/8_5_the_cochrane_collaborations_tool_for_assessing_ risk_of_bias.htm

19. Park J, Lee Y, Seo H, et al. Risk of Bias Assessment tool for Nonrandomized Studies (RoBANS): development and validation of a new instrument. 19th Cochrane Colloquium; Madrid, 2011. http:// 2011.colloquium.cochrane.org/abstracts/b8o3-risk-bias-assessmenttool-non-randomized-studies-robans-development-and-validation-ne

20. The GRADE Working Group. List of GRADE working group publications and grants. http://www.gradeworkinggroup.org/ (accessed 27 Sep 2015)

21. Mikkelsen M, Husby S, Skov L, et al. A systematic review of types of healthy eating interventions in preschools. Nutr $J$ 2014;13:56

22. Seymour J, Yaroch A, Serdula M, et al. Impact of nutrition environmental interventions on point-of-purchase behavior in adults: a review. Prev Med (Baltim) 2004;39:S108-36.

23. Were WM, Daelmans B, Bhutta Z, et al. Children's health priorities and interventions. BMJ 2015;351:h4300.

24. Webb P, Boyd E, de Pee S, et al. Nutrition in emergencies: do we know what works? Food Policy 2014;49:33-40.

25. Hall A, Blankson B, Shoham J. The impact and effectiveness of emergency nutrition and nutrition-related interventions: a review of published evidence 2004-2010. Emerg Nutr Netw. Published Online First: 2011. http://www.ennonline.net/ impactevidenceemergencynutrition 